\title{
ASYMPTOTIC EXPANSION AND QUADRATURE OF COMPOSITE HIGHLY OSCILLATORY INTEGRALS
}

\author{
ARIEH ISERLES AND DAVID LEVIN
}

\begin{abstract}
We consider in this paper asymptotic and numerical aspects of highly oscillatory integrals of the form $\int_{a}^{b} f(x) g(\sin [\omega \theta(x)]) \mathrm{d} x$, where $\omega \gg 1$. Such integrals occur in the simulation of electronic circuits, but they are also of independent mathematical interest.

The integral is expanded in asymptotic series in inverse powers of $\omega$. This expansion clarifies the behaviour for large $\omega$ and also provides a powerful means to design effective computational algorithms. In particular, we introduce and analyse Filon-type methods for this integral.
\end{abstract}

\section{INTRODUCTION}

The theory of highly oscillatory integrals is a mature subject and our theoretical understanding of the behaviour of integrals of the form

$$
\int_{a}^{b} f(x) \mathrm{e}^{\mathrm{i} \omega g(x)} \mathrm{d} x
$$

and their multivariate counterparts is fairly complete Olv74, Ste93, Won01]. In the last few years this has been complemented by a comprehensive understanding of the numerical quadrature of such integrals by a range of methods: Filon-type [N04, IN05, Levin-type [Lev96, Olv06, and numerical steepest descent HV06]. It is however in the nature of mathematical research that, no sooner than we declare a theory 'complete', a new application comes to light, provides a counterexample, and challenges our understanding of the subject.

A major stumbling block in the simulation of electronic circuits is the resolution of high frequency modulated signals. An important example is the modulation of a diode rectifier circuit, which is modelled by a nonlinear ordinary differential equation of the form

$$
C v^{\prime}=-\frac{v}{R}+I_{0}\left[\mathrm{e}^{b(t)-v}-1\right], \quad t \geq 0, \quad v(0)=v_{0},
$$

where $C$ is the capacitance, $R$ is the resistance, $I_{0}$ is the diode inverse bias current, $b(t)$ is the input signal and the unknown $v$ is the voltage [DCB05]. Analogue modulation of (1.2) is associated with the input signal $b(t)=\kappa \sin (\omega t)$, where $\omega \gg 1$. An effective numerical solution of (1.2) with this value of $b$, using waveform relaxation, requires efficient computation of integrals of the form

$$
\int_{a}^{b} f(x) \mathrm{e}^{\kappa \sin (\omega x)} \mathrm{d} x
$$

Received by the editor October 30, 2008 and, in revised form, August 21, 2009.

2010 Mathematics Subject Classification. Primary 65D30; Secondary 41A55.

(C)2010 American Mathematical Society 
$\mathrm{CDI}^{+} 09$. The integral (1.3) does not fit into the classical pattern (1.1) of highly oscillatory integrals or of its generalisations, whereby $\exp [\mathrm{i} \omega g(x)]$ is replaced by a suitable fundamental solution of a linear ordinary differential equation [Olv08] and, indeed, as will transpire shortly, its behaviour is altogether different. In particular, while (for $-\infty<a<b<\infty$ ) the integral (1.1) behaves like $\mathcal{O}\left(\omega^{-1 /(r+1)}\right)$ for $\omega \rightarrow \infty$, where $r$ is the highest degree of a stationary point $\left(r=0\right.$ if $g^{\prime} \neq 0$ in the closed interval), the integral (1.3) is $\mathcal{O}(1)$ for $\omega \rightarrow \infty$.

It is possible to analyse (and, indeed, compute) the integral (1.3) by exploiting a serendipitous identity,

$$
\mathrm{e}^{\kappa \sin \theta}=I_{0}(\kappa)+2 \sum_{m=0}^{\infty}(-1)^{m} I_{m}(\kappa) \sin [(2 m+1) \theta]+2 \sum_{m=1}^{\infty}(-1)^{m} I_{2 m}(\kappa) \cos (2 m \theta),
$$

where $I_{m}$ is the $m$ th modified Bessel function AS64, p. 376], and this has been already done in [CDI09]. The purpose of the present paper is more ambitious, namely to consider integrals of the form

$$
\boldsymbol{I}[f, g]=\int_{a}^{b} f(x) g(\sin (\omega x)) \mathrm{d} x, \quad \omega \gg 1,
$$

where $f \in \mathrm{C}^{\infty}[a, b]$ and $g$ is analytic in the disc $|z|\langle r$ for some $r>1$ and $\omega \gg 1$. In that case we no longer enjoy the benefits of serendipity and need to work out the asymptotic behaviour of (1.4) from basic premises.

An interesting generalisation of (1.4) is to the integral

$$
\boldsymbol{I}[f, g, \theta]=\int_{a}^{b} f(x) g(\sin [\omega \theta(x)]) \mathrm{d} x,
$$

where $\left.\theta \in \mathrm{C}^{\infty}[a, b] 1\right]$ Provided that $\theta^{\prime} \neq 0$ in $[a, b]$, our analysis can be easily extended to this setting. Matters are more difficult when $\theta^{\prime}$ is allowed to vanish, a situation similar to the presence of stationary points in classical theory. In that case we indicate how to obtain an expansion using an approach similar to [N05.

In Section 2 we expand (1.4) into asymptotic series in inverse powers of $\omega$, while Section 3 is devoted to the efficient numerical quadrature of this integral by means of a variant of a Filon-type method.

We note in passing that our approach is applicable also to the integral

$$
\int_{a}^{b} f(x) g(\cos (\omega x)) \mathrm{d} x, \quad \omega \gg 1 .
$$

Since an identical methodology applies and the results are similar, we do not dwell further on this.

\section{The ASYMPtotic EXPANSION OF THE INTEGRAL}

2.1. The basic expansion. Expanding the analytic function $g$ into Taylor series, we have

$$
\boldsymbol{I}[f, g]=\sum_{n=0}^{\infty} \frac{g^{(n)}(0)}{n !} \boldsymbol{S}_{n}[f]
$$

\footnotetext{
${ }^{1}$ Here and elsewhere the $\mathrm{C}^{\infty}[a, b]$ assumption can be relaxed and replaced by $\mathrm{C}^{\nu}[a, b]$ for some $\nu \geq 1$, except that in that case only a finite number of leading terms in our expansions are valid.
} 
where

$$
\boldsymbol{S}_{n}[f]=\int_{a}^{b} f(x) \sin ^{n}(\omega x) \mathrm{d} x, \quad n \in \mathbb{Z}_{+} .
$$

Thus, our first goal is to expand each functional $\boldsymbol{S}_{n}[f]$ asymptotically in $\boldsymbol{\omega}$.

Noting that

$$
\begin{aligned}
\boldsymbol{S}_{n}[f] & =\frac{1}{(2 \mathrm{i})^{n}} \int_{a}^{b} f(x)\left(\mathrm{e}^{\mathrm{i} \omega x}-\mathrm{e}^{-\mathrm{i} \omega x}\right)^{n} \mathrm{~d} x \\
& =\frac{1}{(2 \mathrm{i})^{n}} \sum_{m=0}^{n}(-1)^{n-m}\left(\begin{array}{c}
n \\
m
\end{array}\right) \int_{a}^{b} f(x) \mathrm{e}^{\mathrm{i} \omega(2 m-n) x} \mathrm{~d} x
\end{aligned}
$$

we need to distinguish between even and odd values of $n$. This leads to calculations which, although lengthy, are quite elementary.

For even $n$ we have

$$
\begin{aligned}
& \boldsymbol{S}_{2 n}[f]=\frac{(-1)^{n}}{4^{n}}\left\{(-1)^{n}\left(\begin{array}{c}
2 n \\
n
\end{array}\right) \int_{a}^{b} f(x) \mathrm{d} x\right. \\
& \left.+\sum_{m=0}^{n-1}(-1)^{m}\left(\begin{array}{c}
2 n \\
m
\end{array}\right) \int_{a}^{b} f(x)\left[\mathrm{e}^{2 \mathrm{i} \omega(n-m) x}+\mathrm{e}^{-2 \mathrm{i} \omega(n-m) x}\right] \mathrm{d} x\right\} \\
& \sim \frac{1}{4^{n}}\left(\begin{array}{c}
2 n \\
n
\end{array}\right) \int_{a}^{b} f(x) \mathrm{d} x-\frac{(-1)^{n}}{4^{n}} \sum_{m=0}^{n-1}(-1)^{m}\left(\begin{array}{c}
2 n \\
m
\end{array}\right) \sum_{k=0}^{\infty} \frac{1}{(-\mathrm{i} \omega)^{k+1}} \\
& \times\left\{f^{(k)}(b)\left[\frac{\mathrm{e}^{2 \mathrm{i} \omega(n-m) b}}{(2 n-2 m)^{k+1}}+\frac{\mathrm{e}^{-2 \mathrm{i} \omega(n-m) b}}{(2 m-2 n)^{k+1}}\right]\right. \\
& \left.-f^{(k)}(a)\left[\frac{\mathrm{e}^{2 \mathrm{i} \omega(n-m) a}}{(2 n-2 m)^{k+1}}+\frac{\mathrm{e}^{-2 \mathrm{i} \omega(n-m) a}}{(2 m-2 n)^{k+1}}\right]\right\} \\
& =\frac{1}{4^{n}}\left(\begin{array}{c}
2 n \\
n
\end{array}\right) \int_{a}^{b} f(x) \mathrm{d} x-\frac{(-1)^{n}}{4^{n}} \sum_{m=0}^{n-1}(-1)^{m}\left(\begin{array}{c}
2 n \\
m
\end{array}\right) \sum_{k=0}^{\infty} \frac{1}{[-2 \mathrm{i} \omega(n-m)]^{k+1}} \\
& \times\left\{f^{(k)}(b)\left[\mathrm{e}^{2 \mathrm{i} \omega(n-m) b}-(-1)^{k} \mathrm{e}^{-2 \mathrm{i} \omega(n-m) b}\right]\right. \\
& \left.-f^{(k)}(a)\left[e^{2 \mathrm{i} \omega(n-m) a}-(-1)^{k} \mathrm{e}^{-2 \mathrm{i} \omega(n-m) a}\right]\right\} \\
& =\frac{1}{4^{n}}\left(\begin{array}{c}
2 n \\
n
\end{array}\right) \int_{a}^{b} f(x) \mathrm{d} x+\frac{2(-1)^{n}}{4^{n}} \sum_{m=0}^{n-1}(-1)^{m}\left(\begin{array}{c}
2 n \\
m
\end{array}\right) \\
& \times\left\{\sum _ { k = 0 } ^ { \infty } \frac { ( - 1 ) ^ { k } } { [ 2 \omega ( n - m ) ] ^ { 2 k + 1 } } \left[f^{(2 k)}(b) \sin 2 \omega(n-m) b\right.\right. \\
& \left.-f^{(2 k)}(a) \sin 2 \omega(n-m) a\right] \\
& +\sum_{k=0}^{\infty} \frac{(-1)^{k}}{[2 \omega(n-m)]^{2 k+2}}\left[f^{(2 k+1)}(b) \cos 2 \omega(n-m) b\right. \\
& \left.\left.-f^{(2 k+1)}(a) \cos 2 \omega(n-m) a\right]\right\}
\end{aligned}
$$




$$
\begin{aligned}
&= \frac{1}{4^{n}}\left(\begin{array}{c}
2 n \\
n
\end{array}\right) \int_{a}^{b} f(x) \mathrm{d} x \\
&+\frac{2(-1)^{n}}{4^{n}} \sum_{k=0}^{\infty} \frac{(-1)^{k}}{\omega^{2 k+1}} f^{(2 k)}(b) \sum_{m=0}^{n-1}(-1)^{m}\left(\begin{array}{c}
2 n \\
m
\end{array}\right) \frac{\sin 2 \omega(n-m) b}{[2(n-m)]^{2 k+1}} \\
&-\frac{2(-1)^{n}}{4^{n}} \sum_{k=0}^{\infty} \frac{(-1)^{k}}{\omega^{2 k+1}} f^{(2 k)}(a) \sum_{m=0}^{n-1}(-1)^{m}\left(\begin{array}{c}
2 n \\
m
\end{array}\right) \frac{\sin 2 \omega(n-m) a}{[2(n-m)]^{2 k+1}} \\
&+\frac{2(-1)^{n}}{4^{n}} \sum_{k=0}^{\infty} \frac{(-1)^{k}}{\omega^{2 k+2}} f^{(2 k+1)}(b) \sum_{m=0}^{n-1}(-1)^{m}\left(\begin{array}{c}
2 n \\
m
\end{array}\right) \frac{\cos 2 \omega(n-m) b}{[2(n-m)]^{2 k+2}} \\
&-\frac{2(-1)^{n}}{4^{n}} \sum_{k=0}^{\infty} \frac{(-1)^{k}}{\omega^{2 k+2}} f^{(2 k+1)}(a) \sum_{m=0}^{n-1}(-1)^{m}\left(\begin{array}{c}
2 n \\
m
\end{array}\right) \frac{\cos 2 \omega(n-m) a}{[2(n-m)]^{2 k+2}} \\
& \frac{1}{4^{n}}\left(\begin{array}{c}
2 n \\
n
\end{array}\right) \int_{a}^{b} f(x) \mathrm{d} x+\frac{2}{4^{n}} \sum_{k=0}^{\infty} \frac{(-1)^{k}}{\omega^{2 k+1}} f^{(2 k)}(b) \\
&-\frac{2}{4^{n}} \sum_{k=0}^{\infty} \frac{(-1)^{k}}{\omega^{2 k+1}} f^{(2 k)}(a) \sum_{m=1}^{n}(-1)^{m}\left(\begin{array}{c}
2 n \\
n-m
\end{array}\right) \frac{\sin 2 \omega m a}{(2 m)^{2 k+1}} \\
&+\frac{2}{4^{n}} \sum_{k=0}^{\infty} \frac{(-1)^{k}}{\omega^{2 k+2}} f^{(2 k+1)}(b) \sum_{m=1}^{n}(-1)^{m}\left(\begin{array}{c}
2 n \\
n-m
\end{array}\right) \frac{\cos 2 \omega m b}{(2 m)^{2 k+2}} \\
&-\frac{2}{4^{n}} \sum_{k=0}^{\infty} \frac{(-1)^{k}}{\omega^{2 k+2}} f^{(2 k+1)}(a) \sum_{m=1}^{n}(-1)^{m}\left(\begin{array}{c}
2 n \\
n-m
\end{array}\right) \frac{\cos 2 \omega m a}{(2 m)^{2 k+2}} . \\
& n-m
\end{aligned}
$$

Thus, we have expanded $\boldsymbol{S}_{2 n}[f]$ in inverse powers of $\omega$. Similarly to the familiar expansions of integrals (1.1) in the absence of stationary points, the coefficients of the expansion depend on $f$ and its derivatives at the endpoints. However, an important difference is that, while the integral in (1.1) tends to zero for $\omega \rightarrow \infty$, we have

$$
\boldsymbol{S}_{2 n}[f] \sim \frac{1}{4^{n}}\left(\begin{array}{c}
2 n \\
n
\end{array}\right) \int_{a}^{b} f(x) \mathrm{d} x, \quad \omega \rightarrow \infty .
$$

Note that it is sufficient that $f \in \mathrm{C}[a, b]$ for this to be true.

The expansion for odd index is similar, i.e.,

$$
\begin{aligned}
& \boldsymbol{S}_{2 n+1}[f]=-\frac{1}{(2 \mathrm{i})^{2 n+1}} \sum_{m=0}^{2 n+1}(-1)^{m}\left(\begin{array}{c}
2 n+1 \\
m
\end{array}\right) \int_{a}^{b} f(x) \mathrm{e}^{\mathrm{i} \omega(2 m-2 n-1) x} \mathrm{~d} x \\
&=-\frac{(-1)^{n} \mathrm{i}}{2 \cdot 4^{n}} \sum_{m=0}^{n}(-1)^{m}\left(\begin{array}{c}
2 n+1 \\
m
\end{array}\right) \int_{a}^{b} f(x)\left[\mathrm{e}^{\mathrm{i} \omega(2 n-2 m+1) x}\right. \\
&\left.-\mathrm{e}^{-\mathrm{i} \omega(2 n-2 m+1) x}\right] \mathrm{d} x ;
\end{aligned}
$$


hence

$$
\begin{aligned}
& \boldsymbol{S}_{2 n+1}[f] \sim \frac{(-1)^{n} \mathrm{i}}{2 \cdot 4^{n}} \sum_{m=0}^{n}(-1)^{m}\left(\begin{array}{c}
2 n+1 \\
m
\end{array}\right) \sum_{k=0}^{\infty} \frac{1}{(-\mathrm{i} \omega)^{k+1}} \\
& \times\left\{f^{(k)}(b)\left[\frac{\mathrm{e}^{\mathrm{i} \omega(2 n-2 m+1) b}}{(2 n-2 m+1)^{k+1}}+(-1)^{k} \frac{\mathrm{e}^{-\mathrm{i} \omega(2 n-2 m+1) b}}{(2 n-2 m+1)^{k+1}}\right]\right. \\
& \left.-f^{(k)}(a)\left[\frac{\mathrm{e}^{\mathrm{i} \omega(2 n-2 m+1) a}}{(2 n-2 m+1)^{k+1}}+(-1)^{k} \frac{\mathrm{e}^{-\mathrm{i} \omega(2 n-2 m+1) a}}{(2 n-2 m+1)^{k+1}}\right]\right\} \\
& =\frac{(-1)^{n}}{4^{n}} \sum_{m=0}^{n}(-1)^{m}\left(\begin{array}{c}
2 n+1 \\
m
\end{array}\right)\left\{-\sum_{k=0}^{\infty} \frac{(-1)^{k}}{[\omega(2 n-2 m+1)]^{2 k+1}}\right. \\
& \times\left[f^{(2 k)}(b) \cos \omega(2 n-2 m+1) b-f^{(2 k)}(a) \cos \omega(2 n-2 m+1) a\right] \\
& +\sum_{k=0}^{\infty} \frac{(-1)^{k}}{[\omega(2 n-2 m+1)]^{2 k+2}}\left[f^{(2 k+1)}(b) \sin \omega(2 n-2 m+1) b\right. \\
& \left.\left.-f^{(2 k+1)}(a) \sin \omega(2 n-2 m+1) a\right]\right\} \\
& =\frac{(-1)^{n}}{4^{n}}\left[-\sum_{k=0}^{\infty} \frac{(-1)^{k}}{\omega^{2 k+1}} f^{(2 k)}(b) \sum_{m=0}^{n}(-1)^{m}\left(\begin{array}{c}
2 n+1 \\
m
\end{array}\right)\right. \\
& \times \frac{\cos \omega(2 n-2 m+1) b}{(2 n-2 m+1)^{2 k+1}} \\
& +\sum_{k=0}^{\infty} \frac{(-1)^{k}}{\omega^{2 k+1}} f^{(2 k)}(a) \sum_{m=0}^{n}(-1)^{m}\left(\begin{array}{c}
2 n+1 \\
m
\end{array}\right) \frac{\cos \omega(2 n-2 m+1) a}{(2 n-2 m+1)^{2 k+1}} \\
& +\sum_{k=0}^{\infty} \frac{(-1)^{k}}{\omega^{2 k+2}} f^{(2 k+1)}(b) \sum_{m=0}^{n}(-1)^{m}\left(\begin{array}{c}
2 n+1 \\
m
\end{array}\right) \frac{\sin \omega(2 n-2 m+1) b}{(2 n-2 m+1)^{2 k+2}} \\
& \left.-\sum_{k=0}^{\infty} \frac{(-1)^{k}}{\omega^{2 k+2}} f^{(2 k+1)}(a) \sum_{m=0}^{n}(-1)^{m}\left(\begin{array}{c}
2 n+1 \\
m
\end{array}\right) \frac{\sin \omega(2 n-2 m+1) a}{(2 n-2 m+1)^{2 k+2}}\right] \\
& =-\frac{1}{4^{n}} \sum_{k=0}^{\infty} \frac{(-1)^{k}}{\omega^{2 k+1}} f^{(2 k)}(b) \sum_{m=0}^{n}(-1)^{m}\left(\begin{array}{c}
2 n+1 \\
n-m
\end{array}\right) \frac{\cos \omega(2 m+1) b}{(2 m+1)^{2 k+1}} \\
& +\frac{1}{4^{n}} \sum_{k=0}^{\infty} \frac{(-1)^{k}}{\omega^{2 k+1}} f^{(2 k)}(a) \sum_{m=0}^{n}(-1)^{m}\left(\begin{array}{c}
2 n+1 \\
n-m
\end{array}\right) \frac{\cos \omega(2 m+1) a}{(2 m+1)^{2 k+1}} \\
& +\frac{1}{4^{n}} \sum_{k=0}^{\infty} \frac{(-1)^{k}}{\omega^{2 k+2}} f^{(2 k+1)}(b) \sum_{m=0}^{n}(-1)^{m}\left(\begin{array}{c}
2 n+1 \\
n-m
\end{array}\right) \frac{\sin \omega(2 m+1) b}{(2 m+1)^{2 k+2}} \\
& -\frac{1}{4^{n}} \sum_{k=0}^{\infty} \frac{(-1)^{k}}{\omega^{2 k+2}} f^{(2 k+1)}(a) \sum_{m=0}^{n}(-1)^{m}\left(\begin{array}{c}
2 n+1 \\
n-m
\end{array}\right) \frac{\sin \omega(2 m+1) a}{(2 m+1)^{2 k+2}} .
\end{aligned}
$$

We substitute asymptotic expansions of $\boldsymbol{S}_{n}[f]$ for even and odd values of $n$ into (2.1). The outcome is an asymptotic expansion of the integral $\boldsymbol{I}[f, g]$ in inverse 
powers of large parameter $\omega$, i.e.,

$$
\begin{aligned}
& I[f] \sim 2 \sum_{n=0}^{\infty} \frac{g^{(2 n)}(0)}{2^{2 n}}\left[\frac{1}{2} \frac{1}{(n !)^{2}} \int_{a}^{b} f(x) \mathrm{d} x\right. \\
& +\sum_{k=0}^{\infty} \frac{(-1)^{k}}{\omega^{2 k+1}} f^{(2 k)}(b) \sum_{m=1}^{n} \frac{(-1)^{m}}{(n-m) !(n+m) !} \frac{\sin 2 \omega m b}{(2 m)^{2 k+1}} \\
& -\sum_{k=0}^{\infty} \frac{(-1)^{k}}{\omega^{2 k+1}} f^{(2 k)}(a) \sum_{m=1}^{n} \frac{(-1)^{m}}{(n-m) !(n+m) !} \frac{\sin 2 \omega m a}{(2 m)^{2 k+1}} \\
& +\sum_{k=0}^{\infty} \frac{(-1)^{k}}{\omega^{2 k+2}} f^{(2 k+1)}(b) \sum_{m=1}^{n} \frac{(-1)^{m}}{(n-m) !(n+m) !} \frac{\cos 2 \omega m b}{(2 m)^{2 k+2}} \\
& \left.-\sum_{k=0}^{\infty} \frac{(-1)^{k}}{\omega^{2 k+2}} f^{(2 k+1)}(a) \sum_{m=1}^{n} \frac{(-1)^{m}}{(n-m) !(n+m) !} \frac{\cos 2 \omega m a}{(2 m)^{2 k+2}}\right] \\
& +2 \sum_{n=0}^{\infty} \frac{g^{(2 n+1)}(0)}{2^{2 n+1}}\left[-\sum_{k=0}^{\infty} \frac{(-1)^{k}}{\omega^{2 k+1}} f^{(2 k)}(b) \sum_{m=0}^{n} \frac{(-1)^{m}}{(n-m) !(n+m+1) !}\right. \\
& \times \frac{\cos \omega(2 m+1) b}{(2 m+1)^{2 k+1}} \\
& +\sum_{k=0}^{\infty} \frac{(-1)^{k}}{\omega^{2 k+1}} f^{(2 k)}(a) \sum_{m=0}^{n} \frac{(-1)^{m}}{(n-m) !(n+m+1) !} \frac{\cos \omega(2 m+1) a}{(2 m+1)^{2 k+1}} \\
& +\sum_{k=0}^{\infty} \frac{(-1)^{k}}{\omega^{2 k+2}} f^{(2 k+1)}(b) \sum_{m=0}^{n} \frac{(-1)^{m}}{(n-m) !(n+m+1) !} \frac{\sin \omega(2 m+1) b}{(2 m+1)^{2 k+2}} \\
& \left.-\sum_{k=0}^{\infty} \frac{(-1)^{k}}{\omega^{2 k+2}} f^{(2 k+1)}(a) \sum_{m=0}^{n} \frac{(-1)^{m}}{(n-m) !(n+m+1) !} \frac{\sin \omega(2 m+1) a}{(2 m+1)^{2 k+2}}\right] \\
& =\sum_{n=0}^{\infty} \frac{g^{(2 n)}(0)}{(n !)^{2}} \frac{1}{2^{2 n}} \int_{a}^{b} f(x) \mathrm{d} x \\
& +2 \sum_{k=0}^{\infty} \frac{(-1)^{k}}{\omega^{2 k+1}} f^{(2 k)}(b) \sum_{m=1}^{\infty} \frac{(-1)^{m}}{(2 m)^{2 k+1}} \frac{\sin 2 \omega m b}{2^{2 m}} \sum_{n=0}^{\infty} \frac{g^{(2 m+2 n)}(0)}{n !(2 m+n) !} \frac{1}{4^{n}} \\
& -2 \sum_{k=0}^{\infty} \frac{(-1)^{k}}{\omega^{2 k+1}} f^{(2 k)}(a) \sum_{m=1}^{\infty} \frac{(-1)^{m}}{(2 m)^{2 k+1}} \frac{\sin 2 \omega m a}{2^{2 m}} \sum_{n=0}^{\infty} \frac{g^{(2 m+2 n)}(0)}{n !(2 m+n) !} \frac{1}{4^{n}} \\
& -2 \sum_{k=0}^{\infty} \frac{(-1)^{k}}{\omega^{2 k+1}} f^{(2 k)}(b) \sum_{m=0}^{\infty} \frac{(-1)^{m}}{(2 m+1)^{2 k+1}} \frac{\cos \omega(2 m+1) b}{2^{2 m+1}} \sum_{n=0}^{\infty} \frac{g^{(2 m+2 n+1)}(0)}{n !(2 m+1+n) !} \frac{1}{4^{n}} \\
& +2 \sum_{k=0}^{\infty} \frac{(-1)^{k}}{\omega^{2 k+1}} f^{(2 k)}(a) \sum_{m=0}^{\infty} \frac{(-1)^{m}}{(2 m+1)^{2 k+1}} \frac{\cos \omega(2 m+1) a}{2^{2 m+1}} \sum_{n=0}^{\infty} \frac{g^{(2 m+2 n+1)}(0)}{n !(2 m+1+n) !} \frac{1}{4^{n}} \\
& +2 \sum_{k=0}^{\infty} \frac{(-1)^{k}}{\omega^{2 k+2}} f^{(2 k+1)}(b) \sum_{m=1}^{\infty} \frac{(-1)^{m}}{(2 m)^{2 k+2}} \frac{\cos 2 \omega m b}{2^{2 m}} \sum_{n=0}^{\infty} \frac{g^{(2 m+2 n)}(0)}{n !(2 m+n) !} \frac{1}{4^{n}} \\
& -2 \sum_{k=0}^{\infty} \frac{(-1)^{k}}{\omega^{2 k+2}} f^{(2 k+1)}(a) \sum_{m=1}^{\infty} \frac{(-1)^{m}}{(2 m)^{2 k+2}} \frac{\cos 2 \omega m a}{2^{2 m}} \sum_{n=0}^{\infty} \frac{g^{(2 m+2 n)}(0)}{n !(2 m+n) !} \frac{1}{4^{n}}
\end{aligned}
$$




$$
\begin{aligned}
& +2 \sum_{k=0}^{\infty} \frac{(-1)^{k}}{\omega^{2 k+2}} f^{(2 k+1)}(b) \sum_{m=0}^{\infty} \frac{(-1)^{m}}{(2 m+1)^{2 k+2}} \frac{\sin \omega(2 m+1) b}{2^{2 m+1}} \sum_{n=0}^{\infty} \frac{g^{(2 m+2 n+1)}(0)}{n !(2 m+1+n) !} \frac{1}{4^{n}} \\
& -2 \sum_{k=0}^{\infty} \frac{(-1)^{k}}{\omega^{2 k+2}} f^{(2 k+1)}(a) \sum_{m=0}^{\infty} \frac{(-1)^{m}}{(2 m+1)^{2 k+2}} \frac{\sin \omega(2 m+1) a}{2^{2 m+1}} \sum_{n=0}^{\infty} \frac{g^{(2 m+2 n+1)}(0)}{n !(2 m+1+n) !} \frac{1}{4^{n}}
\end{aligned}
$$

Let

$$
\rho_{m}=\frac{1}{2^{m-1}} \sum_{n=0}^{\infty} \frac{g^{(m+2 n)}(0)}{n !(m+n) !} \frac{1}{4^{n}}, \quad m \in \mathbb{Z}_{+},
$$

and set for all $k \in \mathbb{Z}_{+}$

$$
\begin{aligned}
& U_{k}(t)=\sum_{m=1}^{\infty} \frac{(-1)^{m}}{(2 m)^{2 k+1}} \rho_{2 m} \sin (2 \omega m t)-\sum_{m=0}^{\infty} \frac{(-1)^{m}}{(2 m+1)^{2 k+1}} \rho_{2 m+1} \cos [\omega(2 m+1) t], \\
& V_{k}(t)=\sum_{m=1}^{\infty} \frac{(-1)^{m}}{(2 m)^{2 k+2}} \rho_{2 m} \cos (2 \omega m t)+\sum_{m=0}^{\infty} \frac{(-1)^{m}}{(2 m+1)^{2 k+2}} \rho_{2 m+1} \sin [\omega(2 m+1) t] .
\end{aligned}
$$

Then

$$
\begin{aligned}
\boldsymbol{I}[f, g] \sim & \frac{1}{2} \rho_{0} \int_{a}^{b} f(x) \mathrm{d} x+\sum_{k=0}^{\infty} \frac{(-1)^{k}}{\omega^{2 k+1}}\left[f^{(2 k)}(b) U_{k}(b)-f^{(2 k)}(a) U_{k}(a)\right] \\
& +\sum_{k=0}^{\infty} \frac{(-1)^{k}}{\omega^{2 k+2}}\left[f^{(2 k+1)}(b) V_{k}(b)-f^{(2 k+1)}(a) V_{k}(a)\right]
\end{aligned}
$$

Note that the function $g$ enters the asymptotic expansion solely through the sequence $\boldsymbol{\rho}=\left\{\rho_{m}\right\}_{m \in \mathbb{Z}_{+}}$, while $f$ contributes both through a nonoscillatory integral and derivatives at the endpoints.

2.2. The sequence $\boldsymbol{\rho}$. In the present subsection we consider the sequence $\boldsymbol{\rho}=$ $\left\{\rho_{m}\right\}_{m \in \mathbb{Z}_{+}}$. Our main concern is with the asymptotic behaviour of the sequence. Examining the functions $U_{k}$ and $V_{k}$, it is clear that geometric decay, $\rho_{m} \approx c \tilde{\rho}^{m}$ for some $\tilde{\rho} \in(0,1)$, is sufficient for their existence and boundedness for all $k \in \mathbb{Z}_{+}$, a sine qua non for the applicability of the expansion (2.3).

We commence with three detailed examples. First, we let $g(x)=\mathrm{e}^{\kappa x}, \kappa \in \mathbb{R} \backslash\{0\}$; therefore consider the integral (1.3). This yields

$$
\rho_{m}=2^{1-m} \kappa^{m} \sum_{n=0}^{\infty} \frac{1}{n !(m+n) !}\left(\frac{\kappa}{2}\right)^{2 n}=2 \frac{(\kappa / 2)^{m}}{m !}{ }_{0} F_{1}\left[\begin{array}{l}
-; \\
m+1 ;
\end{array} \frac{\kappa^{2}}{2}\right]=2 I_{m}(\kappa),
$$

where we have used the hypergeometric representation of modified Bessel functions [Rai60, p. 116]. After brief manipulation, we recover the serendipitous asymptotic expansion from CDI09. Note that standard asymptotic expansions of modified Bessel functions for large index demonstrate at once the extraordinarily rapid decay of the sequence $\boldsymbol{\rho}$, because $I_{m}(\kappa)=\mathcal{O}(1 / m !)$.

We next consider the case $g(x)=\sin \kappa x$, where $\kappa \in \mathbb{R} \backslash\{0\}$. Similar algebra confirms that

$$
\rho_{2 m}=0, \quad \rho_{2 m+1}=2(-1)^{m} J_{2 m+1}(\kappa),
$$

where $J_{m}$ is the Bessel function. Again, the sequence $\boldsymbol{\rho}$ decays at a faster-thanexponential speed. 
A more interesting example, of which we will make use in the sequel, is $g(x)=$ $(1-\kappa x)$, where $|\kappa|<1$. Therefore $g^{(n)}(0)=n ! \kappa^{n}$ and



Let

$$
\chi(z)={ }_{2} F_{1}\left[\frac{m+1}{2}, \frac{m+2}{2} ; z\right], \quad|z|<1 .
$$

We use the identity

$$
\frac{1}{(1+x)^{2 a}}{ }_{2} F_{1}\left[\begin{array}{ll}
a, b ; & 4 x \\
2 b ; & (1+x)^{2}
\end{array}\right]={ }_{2} F_{1}\left[\begin{array}{l}
a, a-b+\frac{1}{2} ; \\
b+\frac{1}{2} ;
\end{array}\right]
$$

Rai60, p. 65] with $a=m / 2+1, b=(m+1) / 2$, and $x=(2-t-2 \sqrt{1-t}) / t$. After long, yet elementary, algebraic computation, and bearing in mind that for our values of $a$ and $b$ it is true that

we have



$$
\chi(z)=\frac{1}{\sqrt{1-z}}\left[\frac{2(1-\sqrt{1-z})}{z}\right]^{m}=\frac{1}{\sqrt{1-z}}\left(\frac{2}{1+\sqrt{1-z}}\right)^{m} .
$$

In particular, recalling that $|\kappa|<1$, we deduce

$$
\rho_{m}=\frac{1}{\sqrt{1-\kappa^{2} / 4}}\left(\frac{\kappa}{1+\sqrt{1-\kappa^{2} / 4}}\right)^{m}, \quad m \in \mathbb{Z}_{+},
$$

an exponential decay.

Note that the above identity from Rai60] is true only when $|x|<1$ and $4|x|<$ $|1+x|^{2}$. This is not a problem, because we can extend our explicit form of $\chi$ elsewhere by analytic continuation. The only subtle point about it is that we might need to change the sign of the square root once we cross the branch cut $\operatorname{Re} z=0$.

Theorem 1. Given any analytic function $g$ with radius of convergence $r>1$, it is true that $\lim _{m \rightarrow \infty} \rho_{m}=0$. Moreover, if $r<\infty$, then $\rho_{m}=o\left(r^{-2 m}\right)$, while if $g$ is entire, i.e., $r=+\infty$, then $\boldsymbol{\rho}$ decays faster than a reciprocal of a polynomial.

Proof. Applying the Cauchy test to the Taylor expansion of $g$ about the origin,

$$
\frac{1}{r}=\left|\frac{g^{(n)}(0)}{n !}\right|^{1 / n}=\frac{\mathrm{e}\left|g^{(n)}(0)\right|^{1 / n}}{n}+o(1), \quad n \rightarrow \infty,
$$

where we have used the Stirling formula to approximate the factorial. Next, we apply the Cauchy test to the definition of $\rho_{m}$ : convergence is equivalent to

$$
1>\limsup _{n \rightarrow \infty}\left|\frac{g^{(m+2 n)}(0)}{n !(m+n) ! 4^{n}}\right|^{1 / n}=\limsup _{n \rightarrow \infty} \frac{\left[\left|g^{(m+2 n)}(0)\right|^{1 /(m+2 n)}\right]^{2+m / n}}{4(n !)^{1 / n}[(m+n) !]^{1 / n}} .
$$

Using again the Stirling formula and exploiting the fact that

$$
\limsup _{n \rightarrow \infty} \frac{\left|g^{(m+2 n)}(0)\right|^{1 /(m+2 n)}}{m+2 n} \leq \limsup _{n \rightarrow \infty} \frac{\left|g^{(n)}(0)\right|^{1 / n}}{n}=\frac{1}{\mathrm{er}},
$$

we deduce that the limsup on the right is $r^{-2}<1$; hence we have convergence. 
The assertion on speed of convergence follows at once from the Cauchy criterion.

Noting the importance of Theorem 1 in justifying the validity of the asymptotic expansion (2.3), we also observe an important potential shortcoming of the definition (2.2). Numerical computation of high derivatives of a function $g$, its smoothness notwithstanding, is a notoriously ill-conditioned problem. We will return to this issue in the sequel, but at present we generalise from our examples and provide an explicit formula for a very important subset of functions $g$.

Recall that a generalised hypergeometric function is

$$
{ }_{p} F_{q}\left[\begin{array}{c}
\alpha_{1}, \alpha_{2}, \ldots, \alpha_{p} ; \\
\beta_{1}, \beta_{2}, \ldots, \beta_{q} ;
\end{array}\right]=\sum_{n=0}^{\infty} \frac{1}{n !} \frac{\prod_{i=1}^{p}\left(\alpha_{i}\right)_{n}}{\prod_{i=1}^{q}\left(\beta_{i}\right)_{n}} x^{n},
$$

where the Pochhammer symbol $(z)_{n}$ is defined recursively, $(z)_{0}=1$ and $(z)_{n}=$ $(z)_{n-1}(z+n-1), n \in \mathbb{N}$ Rai60]. The parameters $\alpha_{i}$ and $\beta_{i}$ are arbitrary complex numbers, except that the $\beta_{i}$ 's can be neither zero nor negative integers. Not just our three examples but many other important functions in applied mathematics and theoretical physics can be written using hypergeometric functions.

Let us assume that $a=-1, b=1$, and

$$
g(x)={ }_{p} F_{q}\left[\begin{array}{c}
\alpha_{1}, \alpha_{2}, \ldots, \alpha_{p} ; \kappa x \\
\beta_{1}, \beta_{2}, \ldots, \beta_{q} ; \kappa
\end{array}\right] .
$$

To assure ourselves of analyticity in $[-1,1]$, we require $q+1 \geq p$ and that if $q+1=p$, then $|\kappa|<1$. Since

$$
g^{(n)}(0)=\frac{\prod_{i=1}^{p}\left(\alpha_{i}\right)_{n}}{\prod_{i=1}^{q}\left(\beta_{i}\right)_{n}} \kappa^{n}
$$

substitution in (2.2) yields

$$
\rho_{m}=\frac{1}{2^{m-1}} \sum_{n=0}^{\infty} \frac{1}{n !(m+n) !} \frac{\prod_{i=1}^{p}\left(\alpha_{i}\right)_{m+2 n}}{\prod_{i=1}^{q}\left(\beta_{i}\right)_{m+2 n}} \kappa^{m+2 n} .
$$

But

$$
(\gamma)_{m+2 n}=(\gamma)_{m}(\gamma+m)_{2 n}=4^{n}(\gamma)_{m}\left(\frac{\gamma+m}{2}\right)_{n}\left(\frac{\gamma+m+1}{2}\right)_{n}, \quad \gamma \in \mathbb{C}, \quad m, n \in \mathbb{Z}_{+}
$$

therefore

$$
\begin{aligned}
\rho_{m} & =2 \frac{\prod_{i=1}^{p}\left(\alpha_{i}\right)_{m}}{m ! \prod_{i=1}^{q}\left(\beta_{i}\right)_{m}}\left(\frac{\kappa}{2}\right)^{m} \sum_{n=0}^{\infty} \frac{\prod_{i=1}^{p}\left(\frac{\alpha_{i}+m}{2}\right)_{n}\left(\frac{\alpha_{i}+m+1}{2}\right)_{n}}{n !(m+1)_{n} \prod_{i=1}^{q}\left(\frac{\beta_{i}+m}{2}\right)_{n}\left(\frac{\beta_{i}+m+1}{2}\right)_{n}} \frac{\kappa^{2 n}}{4^{(1+q-p) n}} \\
& =2 \frac{\prod_{i=1}^{p}\left(\alpha_{i}\right)_{m}}{m ! \prod_{i=1}^{q}\left(\beta_{j}\right)_{m}}\left(\frac{\kappa}{2}\right)^{m}{ }_{2 p} F_{2 q+1}\left[\begin{array}{c}
\tilde{\alpha}_{1}, \tilde{\alpha}_{2}, \ldots, \tilde{\alpha}_{2 p}, \\
m+1, \tilde{\beta}_{1}, \tilde{\beta}_{2}, \ldots, \tilde{\beta}_{2 q} ; \frac{\kappa^{2}}{4^{1+q-p}}
\end{array}\right],
\end{aligned}
$$

where

$$
\begin{array}{lll}
\tilde{\alpha}_{i}=\frac{\alpha_{i}+m}{2}, & \tilde{\alpha}_{p+i}=\frac{\alpha_{i}+m+1}{2}, & i=1,2, \ldots, p ; \\
\tilde{\beta}_{i}=\frac{\beta_{i}+m}{2}, & \tilde{\beta}_{q+i}=\frac{\beta_{i}+m+1}{2}, & i=1,2, \ldots, q .
\end{array}
$$

Therefore $\rho_{m}$ itself can be expressed as a generalised hypergeometric function. Note that $q+1 \geq p$ implies that $(2 q+1)+1 \geq 2 p$ and $\rho_{m}$ is analytic in $[-1,1]$ as a function of $\kappa$ (or, for that matter, of $\kappa / 4^{1+q-p}$ ). This is consistent with Theorem 1 . 
The importance of this hypergeometric representation of $\boldsymbol{\rho}$ is that most modern software, e.g., Maple, Mathematica, and Matlab, is well equipped to calculate generalised hypergeometric functions. This avoids the need to calculate high derivatives and provides an effective means to compute $\boldsymbol{\rho}$, hence the asymptotic expansion (2.3), when $g$ is a generalised hypergeometric function.

2.3. A generalised oscillator. Our results can be generalised with ease to the integral $\boldsymbol{I}[f, g, \theta]$ defined in (1.5), provided that $\theta^{\prime} \neq 0$ in $[a, b]$. Letting $t=\theta(x)$, a trivial change of variables results in

$$
\boldsymbol{I}[f, g, \theta]=\int_{\theta(a)}^{\theta(b)} \frac{f\left(\theta^{-1}(t)\right)}{\theta^{\prime}\left(\theta^{-1}(t)\right)} g(\sin \omega t) \mathrm{d} t=\boldsymbol{I}[\tilde{f}, g],
$$

where

Therefore, since

$$
\tilde{f}(t)=\frac{f\left(\theta^{-1}(t)\right)}{\theta^{\prime}\left(\theta^{-1}(t)\right)} .
$$

$$
\int_{\theta(a)}^{\theta(b)} \tilde{f}(t) \mathrm{d} t=\int_{a}^{b} f(x) \mathrm{d} x
$$

we deduce from (2.3) that

$$
\begin{aligned}
\boldsymbol{I}[f, g, \theta] \sim & \frac{1}{2} \rho_{0} \int_{a}^{b} f(x) \mathrm{d} x \\
& +\sum_{k=0}^{\infty} \frac{(-1)^{k}}{\omega^{2 k+1}}\left[\tilde{f}^{(2 k)}(\theta(b)) U_{k}(\theta(b))-\tilde{f}^{(2 k)}(\theta(a)) U_{k}(\theta(a))\right] \\
& +\sum_{k=0}^{\infty} \frac{(-1)^{k}}{\omega^{2 k+2}}\left[\tilde{f}^{(2 k+1)}(\theta(b)) V_{k}(\theta(b))-\tilde{f}^{(2 k+1)}(\theta(a)) V_{k}(\theta(a))\right] .
\end{aligned}
$$

Matters are more complicated in the presence of stationary points, where $\theta^{\prime}$ vanishes. We may assume without loss of generality that $\theta^{\prime}(b)=0$ and $\theta^{\prime}(x) \neq 0$ for $x \in[a, b)$, since any integral (1.5) with $r \geq 1$ stationary points can be written as a sum of $r+1$ integrals with a stationary point at an endpoint and the latter can be assumed at the larger endpoint through possible linear transformation of variables.

The change of variables (2.4) remains valid but the expansion (2.5) is unusable, because $\tilde{f}$ and its derivatives have singularity at $b$. Yet, we can extract from (2.4) an important morsel of information. Thus, suppose that $\theta^{(i)}(b)=0, i=1,2, \ldots, q$, and $\theta^{(q+1)}(b) \neq 0$. We assume in addition, without loss of generality, that $\theta(b)=0$. It is trivial to deduce that $\theta^{\prime}\left(\theta^{-1}(t)\right) \approx c \theta^{q /(q+1)}(x)=c t^{q /(q+1)}, c \neq 0$, as $x \rightarrow b$ for some $c \in \mathbb{C} \backslash\{0\}$. Therefore, changing variables $\zeta=\omega t$, we have

$$
\begin{aligned}
\boldsymbol{I}[f, g, \theta] & \approx c^{-1} \omega^{-1 /(q+1)} \int_{\omega \theta(a)}^{\omega \theta(b)} f\left(\theta^{-1}\left(\frac{\zeta}{\omega}\right)\right) \zeta^{-q /(q+1)} g(\sin \zeta) \mathrm{d} \zeta \\
& \sim \mathcal{O}\left(\omega^{-1 /(q+1)}\right), \quad \omega \rightarrow \infty
\end{aligned}
$$

a result similar to the classical van der Corput lemma for integrals (1.1) Ste93.

To obtain an asymptotic expansion, we take a leaf off IN05. Assume for simplicity that $q=1$; therefore $\theta^{\prime}(b)=0$ and $\theta^{\prime \prime}(b) \neq 0$. We add and subtract $f(b)$, 
i.e.,

$$
\begin{aligned}
\boldsymbol{I}[f, g, \theta] & =f(b) \int_{a}^{b} g(\sin [\omega \theta(x)]) \mathrm{d} x+\int_{a}^{b}[f(x)-f(b)] g(\sin [\omega \theta(x)]) \mathrm{d} x \\
& =f(b) \int_{a}^{b} g(\sin [\omega \theta(x)]) \mathrm{d} x+\int_{\theta(a)}^{\theta(b)} \frac{f\left(\theta^{-1}(t)\right)-f(b)}{\theta^{\prime}\left(\theta^{-1}(t)\right)} g(\sin \omega t) \mathrm{d} t .
\end{aligned}
$$

The function

$$
\breve{f}(x)=\frac{f\left(\theta^{-1}(x)\right)-f(b)}{\theta^{\prime}\left(\theta^{-1}(x)\right)}
$$

has a removable singularity at $\theta(b)$ and is $\mathrm{C}^{\infty}[\theta(a), \theta(b)]$. Therefore we can again use (2.3) to expand

$$
\begin{aligned}
\boldsymbol{I}[f, g, \theta] & \sim f(b) \int_{a}^{b} g(\sin [\omega \theta(x)]) \mathrm{d} x+\frac{1}{2} \rho_{0} \int_{a}^{b}[f(x)-f(b)] \mathrm{d} x \\
& +\sum_{k=0}^{\infty} \frac{(-1)^{k}}{\omega^{2 k+1}}\left[\breve{f}^{(2 k)}(\theta(b)) U_{k}(\theta(b))-\breve{f}^{(2 k)}(\theta(a)) U_{k}(\theta(a))\right] \\
& +\sum_{k=0}^{\infty} \frac{(-1)^{k}}{\omega^{2 k+2}}\left[\breve{f}^{(2 k+1)}(\theta(b)) V_{k}(\theta(b))-\breve{f}^{(2 k+1)}(\theta(a)) V_{k}(\theta(a))\right] .
\end{aligned}
$$

Note two important differences between (2.5) and (2.6). Firstly, in the latter equation there is an additional integral which, in general, is unknown - except that, by our analysis, we know that it is $\sim \mathcal{O}\left(\omega^{-1 / 2}\right)$. Secondly, while each $\tilde{f}^{(k)}(\theta(b))$ can be written as a linear combination of $f^{(i)}(b)$ for $i=0,1, \ldots, k$, evaluating $\breve{f}^{(k)}(\theta(b))$ requires $f^{(i)}(b)$ for $i=0,1, \ldots, 2 k$, roughly twice the data, because the endpoint is a removable singularity.

\section{The QuAdrature of $\boldsymbol{I}[f, g]$}

3.1. Asymptotic method. An obvious approach toward the calculation of the integral $\boldsymbol{I}[f, g]$ is to truncate the asymptotic expansion (2.3). In the terminology of [IN05, the outcome is the asymptotic method

$$
\begin{aligned}
\boldsymbol{A}_{s}[f, g]= & \frac{1}{2} \rho_{0} \int_{a}^{b} f(x) \mathrm{d} x+\sum_{k=0}^{s-1} \frac{(-1)^{k}}{\omega^{2 k+1}}\left[f^{(2 k)}(b) U_{k}(b)-f^{(2 k)}(a) U_{k}(a)\right] \\
& +\sum_{k=0}^{s-1} \frac{(-1)^{k}}{\omega^{2 k+2}}\left[f^{(2 k+1)}(b) V_{k}(b)-f^{(2 k+1)}(a) V_{k}(a)\right],
\end{aligned}
$$

where $s \in \mathbb{N}$ is given. Comparing (2.3) with (3.1) demonstrates at once that

$$
\boldsymbol{A}_{s}[f, g]=\boldsymbol{I}[f, g]+\mathcal{O}\left(\omega^{-2 s-1}\right), \quad \omega \rightarrow \infty .
$$

Therefore, similarly to the numerical theory for classical highly oscillatory integrals (1.1), the more we are willing to invest in computing derivatives of $f$ at the endpoints, the better the accuracy for large $\omega$.

Figure 3.1 displays the scaled error committed by the asymptotic method (3.1), as applied to $f(x)=\mathrm{e}^{x}, g(x)=(2-x)^{-1}$ in the interval $[-1,1]$. For each $\omega \in[0,100]$ we have computed the absolute error $\left|\boldsymbol{A}_{s}[f]-I[f]\right|$ and scaled it by $\omega^{2 s+1}$. The asymptotic formula (3.2) indicates that $\omega^{2 s+1}\left|\boldsymbol{A}_{s}[f]-I[f]\right|$ should be bounded away 


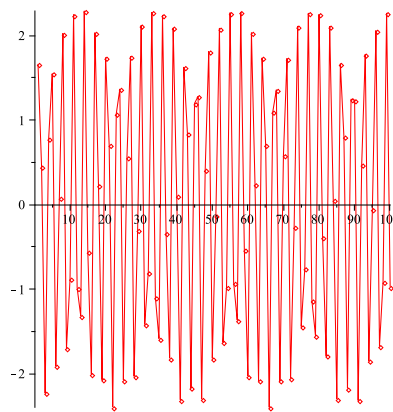

(a)

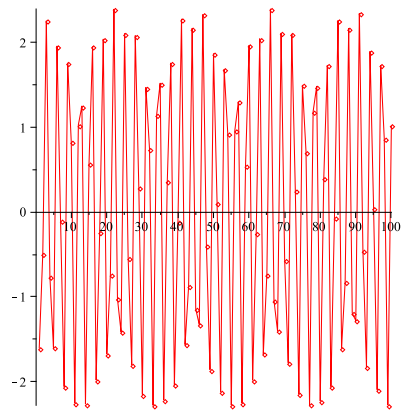

(b)

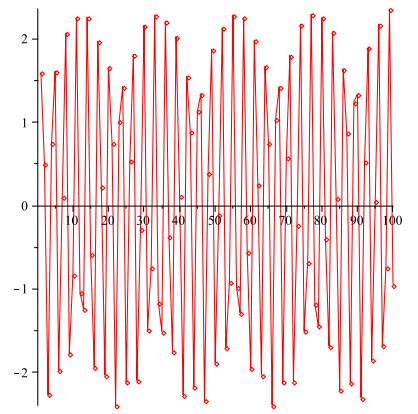

(c)

FiguRE 3.1. Scaled errors $\omega^{2 s+1}\left|\boldsymbol{A}_{s}[f]-I[f]\right|$ for $f(x)=\mathrm{e}^{x}$, $g(x)=(2-x)^{-1},[a, b]=[-1,2]$, and $s=1,2,3$.

from infinity for $\omega \gg 1$ and this is confirmed by the figure. As a matter of fact, the onset of asymptotic behaviour is almost immediate!

An implicit assumption underlying (3.2) is that all the quantities in (3.1) are available in an explicit form. In reality, we require three levels of approximation:

(1) For general $f$ we need to replace the integral by quadrature.

(2) The sequence $\boldsymbol{\rho}$ might need to be approximated.

(3) The functions $U_{k}$ and $V_{k}$ are given as infinite sums and need to be truncated in practical computation.

We address these three approximations in detail.

3.1.1. Computing the nonoscillatory integral. Often the integral of $f$ is known. Otherwise we propose to compute it using an approach from [N08. In order to implement (3.1), we need to compute $f^{(k)}(a)$ and $f^{(k)}(b)$ for $k=0, \ldots, 2 s-1$. The idea is to reuse these values in a Birkhoff quadrature of the form

$$
\int_{a}^{b} f(x) \mathrm{d} x \approx \sum_{k=0}^{2 s-1}\left[w_{k}^{a} f^{(k)}(a)+w_{k}^{b} f^{(k)}(b)\right]
$$

where the weights $w_{k}^{a}$ and $w_{k}^{b}$ are chosen to maximize classical quadrature order. It is easy to verify that (3.3) can be made exact for all polynomials $f$ of order $4 s-1$ and to compute the weights for any $s$ and interval $[a, b]$. For example, for $[a, b]=[-1,1]$ we have $w_{k}^{b}=(-1)^{k} w_{k}^{a}, k=0,1, \ldots, 2 s-1$, and

$$
\begin{array}{rlrl}
s=1: & & \boldsymbol{w}^{a}=\left[1, \frac{1}{3}\right], \quad s=2: \quad \boldsymbol{w}^{a}=\left[1, \frac{3}{7}, \frac{2}{21}, \frac{1}{105}\right], \\
s=3: & \boldsymbol{w}^{a}=\left[1, \frac{5}{11}, \frac{4}{33}, \frac{2}{99}, \frac{1}{495}, \frac{1}{10395}\right] .
\end{array}
$$

One way of improving upon the quadrature (3.3) is to allow the computation of intermediate points,

$$
\int_{a}^{b} f(x) \mathrm{d} x \approx \sum_{k=0}^{2 s-1}\left[w_{k}^{a} f^{(k)}(a)+w_{k}^{b} f^{(k)}(b)\right]+\sum_{l=1}^{\nu} w_{k} f\left(c_{k}\right),
$$

where $c_{k} \in(a, b)$ are given internal nodes. It is easy to verify that for $[a, b]=[-1,1]$ the optimal choice of internal nodes is the zeros of the Jacobi polynomial $P_{\nu}^{(2 s, 2 s)}$ : 
TABLE 1. Errors of different Birkhoff quadrature rules

\begin{tabular}{c|ccccc} 
& $(3.3)$ & $(3.3)$ & $(3.3)$ & $(3.4)$ & $\stackrel{(3.4)}{(3.4)}=3$ \\
$f(x)$ & $s=1$ & $s=2$ & $s=3$ & $s=2, \nu=3$ \\
\hline $\mathrm{e}^{x}$ & $4.77_{-02}$ & $2.11_{-05}$ & $1.47_{-09}$ & $2.05_{-14}$ & $1.11_{-19}$ \\
$(2-x)^{-1}$ & $6.18_{-02}$ & $5.14_{-03}$ & $4.76_{-04}$ & $2.10_{-07}$ & $8.85_{-09}$ \\
$\cos x$ & $4.14_{-02}$ & $1.93_{-05}$ & $1.38_{-09}$ & $1.97_{-14}$ & $1.07_{-19}$
\end{tabular}

the outcome is exact for all polynomials $f$ of degree $4 s-1+2 \nu$. This can be easily extended to arbitrary finite intervals by linear translation.

As an example,

$$
\begin{array}{rlrl}
s=1, \nu & =1: & & \boldsymbol{w}^{a}=\left[\frac{7}{15}, \frac{1}{15}\right], \quad \boldsymbol{w}=\left[\frac{16}{15}\right] ; \\
s=1, \nu=2: & \boldsymbol{w}^{a}=\left[\frac{37}{135}, \frac{1}{45}\right], \quad \boldsymbol{w}=\left[\frac{98}{135}, \frac{98}{135}\right] ; \\
s=2, \nu=1: & \boldsymbol{w}^{a}=\left[\frac{187}{315}, \frac{47}{315}, \frac{2}{105}, \frac{1}{945}\right], \quad \boldsymbol{w}=\left[\frac{256}{315}\right] ; \\
s=2, \nu=2: & \boldsymbol{w}^{a}=\left[\frac{79747}{196875}, \frac{2707}{39375}, \frac{46}{7875}, \frac{1}{4725}\right], \quad \boldsymbol{w}=\left[\frac{117128}{1968751}, \frac{117128}{1968751}\right] ; \\
s=2, \nu=3: & \boldsymbol{w}^{a}=\left[\frac{71353}{240625}, \frac{1761}{48125}, \frac{194}{86625}, \frac{1}{17325}\right], \quad \boldsymbol{w}=\left[\frac{2970344}{6496875}, \frac{1024}{2079}, \frac{2970344}{6496875}\right] .
\end{array}
$$

(The Jacobi polynomial being symmetric with respect to the origin, it remains true that $w_{k}^{b}=(-1)^{k} w_{k}^{a}$.) Note that the weights $w_{k}^{a}$ and $w_{k}$ are all positive. Although we have no formal proof for all $s, \nu \geq 0$, we conjecture that this is true in general. Positivity of weights is important in classical quadrature theory because of its implications for convergence as $\nu \rightarrow \infty$ DR84. We do not pursue this issue further because its importance to the main subject matter of this paper is marginal.

Addition of internal nodes improves drastically the accuracy, as illustrated by Table 1. We present there the absolute error of different quadrature schemes (3.4) and (3.5) for three functions $f$. The dramatic improvement upon the addition of internal points is self evident.

3.1.2. The approximation of $\rho_{m}$. The second step in need of approximation is the computation of the sequence $\boldsymbol{\rho}$ defined in (2.2). We have already seen in Subsection 2.2 that the sequence can be explicitly computed for some functions $g$ and that, once $g$ is a generalised hypergeometric function, each $\rho_{m}$ can be expressed in terms of generalised hypergeometric functions. Such functions can be computed efficiently, often to machine accuracy, with most leading software packages.

Given a general function $g$ which cannot be reduced to a generalised hypergeometric form, an alternative is to interpolate it by a polynomial $\varphi$, say, at points $\vartheta_{1}<\vartheta_{2}<\cdots<\vartheta_{\nu}$ in $[-1,1]$-in other words

$$
g(x) \approx \varphi(x)=\sum_{j=1}^{\nu} \ell_{j}(x) g\left(\vartheta_{j}\right),
$$

where the $\ell_{j}$ 's are cardinal polynomials of Lagrange's interpolation at $\vartheta_{1}, \vartheta_{2}, \ldots, \vartheta_{\nu}$. Other things being equal, the natural choice of $\vartheta_{k}$ 's is as Chebyshev points, since this renders the error $\|g-\varphi\|_{\mathrm{L}_{\infty}[-1,1]}$ small. We then approximate

$$
\rho_{m} \approx \tilde{\rho}_{m}=\frac{1}{2^{m-1}} \sum_{n=0}^{\infty} \frac{\varphi^{(m+2 n)}(0)}{m !(m+n) ! 4^{n}}
$$




$$
=\frac{1}{2^{m-1}} \sum_{j=1}^{\nu} g\left(c_{j}\right) \sum_{n=0}^{\lfloor(\nu-m-1) / 2\rfloor} \frac{\ell_{j}^{(m+2 n)}(0)}{n !(m+n) ! 4^{n}} .
$$

Note that the computation of each $\tilde{\rho}_{m}$ is a finite sum. However, $\tilde{\rho}_{m}=0$ for $m \geq \nu$. This, as well as the need to attain good accuracy, means that we need a sufficiently large value of $\nu$.

An alternative to Lagrangian interpolation is rational approximation. Mindful of the need to ensure analyticity, we consider a function of the form

$$
\psi(x)=\sum_{j=1}^{\nu} \frac{\alpha_{j}}{x-\beta_{j}}
$$

where $\beta_{1}, \beta_{2}, \ldots, \beta_{\nu} \notin[-1,1]$. For example, we can fix the poles $\beta_{j}$ along a perimeter of an ellipse surrounding the interval $[-1,1]$ and determine $a_{1}, a_{2}, \ldots, a_{\nu}$ by imposing interpolation conditions $\psi\left(\vartheta_{j}\right)=g\left(\vartheta_{j}\right)$ for some $\vartheta_{1}, \vartheta_{2}, \ldots, \vartheta_{\nu} \in[a, b]$.

Since

$$
\psi^{(m)}(x)=(-1)^{m} m ! \sum_{j=1}^{\nu} \frac{\alpha_{j}}{\left(x-\beta_{j}\right)^{m+1}},
$$

we have

$$
\psi^{(m)}(0)=-m ! \sum_{j=1}^{\nu} \frac{\alpha_{j}}{\beta_{j}^{m+1}}
$$

and we approximate

$$
\rho_{m} \approx \bar{\rho}_{m}=\frac{1}{2^{m-1}} \sum_{n=0}^{\infty} \frac{\psi^{(m+2 n)}(0)}{n !(m+n) ! 4^{n}}=-\frac{1}{2^{m-1}} \sum_{j=1}^{\nu} \frac{\alpha_{j}}{\beta_{j}} \sum_{n=0}^{\infty} \frac{(m+2 n) !}{n !(m+n) !} \frac{1}{\left(2 \beta_{j}\right)^{m+2 n}} .
$$

Bearing in mind that

$$
(m+n) !=m !(m+1)_{n}
$$

and

$$
(m+2 n) !=4^{n} m !((m+1) / 2)_{m}((m+2) / 2)_{n},
$$

we deduce that

$$
\bar{\rho}_{m}=-\frac{1}{2^{m-1}} \sum_{j=1}^{\nu} \frac{\alpha_{j}}{\beta_{j}^{m+1}} \chi\left(\beta_{j}^{-2}\right)=-\sum_{j=1}^{\nu} \frac{\alpha_{j}}{\sqrt{\beta_{j}^{2}-1}} \frac{1}{\left(\beta_{j}+\sqrt{\beta_{j}^{2}-1}\right)^{m}},
$$

where the function $\chi$ has been defined and discussed in Subsection 2.2. Note that we must be careful in the choice of the sign of $\sqrt{\beta_{j}^{2}-1}$ : since the branch cut is along the line $\operatorname{Re} z=0$, its sign must be the same as the sign of $\operatorname{Re} \beta_{j}$.

Note that $\beta_{j} \notin[-1,1]$ implies $\left|\beta_{j}+\sqrt{\beta_{j}^{2}-1}\right|>1$; therefore the sequence $\{\bar{\rho}\}_{m \in \mathbb{Z}_{+}}$decays geometrically. Similarly to (3.5), the computation of each $\bar{\rho}_{m}$ consists of a finite summation and is probably better conditioned since it does not require the computation of derivatives.

We did not analyse in detail the approximations (3.5) and (3.6) but preliminary computational results indicate the superiority of using rational interpolation.

3.1.3. Computing $U_{k}$ and $V_{k}$. Because of the rapid decay of $\boldsymbol{\rho}$, the four infinite sums required for the computation of $\left\{U_{m}(t), V_{k}(t)\right\}$ converge rapidly. Therefore we can afford to truncate the sums, retaining a relatively small number of terms - the larger $k$, the fewer terms we require. 
3.2. A Filon-type method. Let $a=c_{1}<c_{2}<\cdots<c_{\nu}=b$ be arbitrary quadrature nodes and let $\mu_{1}, \mu_{2}, \ldots, \mu_{\nu} \in \mathbb{N}$ be corresponding multiplicities. Similarly to Filon-type methods for integrals (1.1) in [IN05, we interpolate the function $f$ by a polynomial $\phi$ of degree $N=\sum_{k=1}^{\nu} \mu_{k}-1$,

$$
\phi^{(i)}\left(c_{k}\right)=f^{(i)}\left(c_{k}\right), \quad i=0,1, \ldots, \mu_{k}-1, \quad k=1,2, \ldots, \nu,
$$

and set

$$
\boldsymbol{F}_{r}[f, g]:=\boldsymbol{I}[\phi, g],
$$

where $r=\min \left\{\mu_{1}, \mu_{\nu}\right\}$.

An important feature of the Filon-type method (3.8) is that $\boldsymbol{I}[\varphi, g]$ can be evaluated explicitly, since the asymptotic expansion (2.3) terminates for polynomials, i.e.,

$$
\begin{aligned}
\boldsymbol{F}[\phi, g]= & \frac{1}{2} \rho_{0} \int_{a}^{b} \phi(x) \mathrm{d} x+\sum_{k=0}^{\lfloor N / 2\rfloor} \frac{(-1)^{k}}{\omega^{2 k+1}}\left[\phi^{(2 k)}(b) U_{k}(b)-\phi^{(2 k)}(a) U_{k}(a)\right] \\
& +\sum_{k=0}^{\lfloor(N-1) / 2\rfloor} \frac{(-1)^{k}}{\omega^{2 k+2}}\left[\phi^{(2 k+1)}(b) V_{k}(b)-\phi^{(2 k+1)}(a) V_{k}(a)\right] .
\end{aligned}
$$

Theorem 2. The error of the Filon-type method (3.9) is

$$
\boldsymbol{F}_{r}[f, g]-\boldsymbol{I}[f, g] \sim \boldsymbol{E}[\phi, f]+\mathcal{O}\left(\omega^{-r-1}\right), \quad \omega \rightarrow \infty,
$$

where

$$
\boldsymbol{E}[\phi, f]=\left|\int_{a}^{b}[\phi(x)-f(x)] \mathrm{d} x\right|
$$

is the error of the underlying Birkhoff quadrature.

Proof. Since $\boldsymbol{F}_{r}[f, g]-\boldsymbol{I}[f, g]=\boldsymbol{I}[\phi-f, g]$, the theorem follows at once by substituting $\phi-f$ in place of $f$ in the asymptotic expansion (2.3).

The two above error components, one originating in nonoscillatory quadrature and the other asymptotic, are similar to what we have observed for the asymptotic method and they have already been discussed for the special case $g(x)=\mathrm{e}^{\kappa x}$ in CDI09.

In case $\int_{a}^{b} f(x) \mathrm{d} x$ is known, we replace (3.8) with

$$
\begin{aligned}
\tilde{\boldsymbol{F}}[\phi, g]= & \frac{1}{2} \rho_{0} \int_{a}^{b} f(x) \mathrm{d} x+\sum_{k=0}^{\lfloor N / 2\rfloor} \frac{(-1)^{k}}{\omega^{2 k+1}}\left[\phi^{(2 k)}(b) U_{k}(b)-\phi^{(2 k)}(a) U_{k}(a)\right] \\
& +\sum_{k=0}^{\lfloor(N-1) / 2\rfloor} \frac{(-1)^{k}}{\omega^{2 k+2}}\left[\phi^{(2 k+1)}(b) V_{k}(b)-\phi^{(2 k+1)}(a) V_{k}(a)\right] .
\end{aligned}
$$

It is now trivial to observe that, in place of (3.10), we have

$$
\tilde{\boldsymbol{F}}_{r}[f, g]-\boldsymbol{I}[f, g] \sim \mathcal{O}\left(\omega^{-r-1}\right), \quad \omega \rightarrow \infty,
$$

without any derogatory influence of Birkhoff quadrature error.

In Figure 3.2 we display scaled error for six Filon-type approximations (3.11): note that in our example the nonoscillatory integral is trivial and we computed it explicitly, but, in fairness, it was so in Figure 3.1, and hence we compare like with like. 


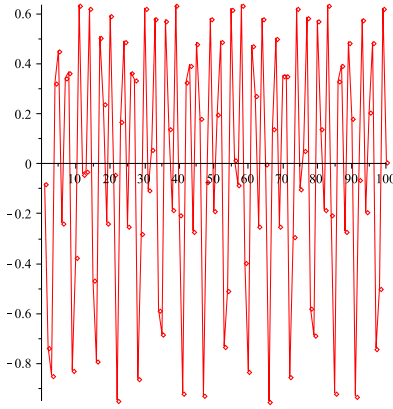

(a)

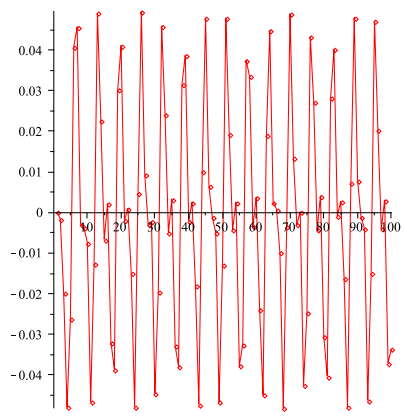

(d)



(b)

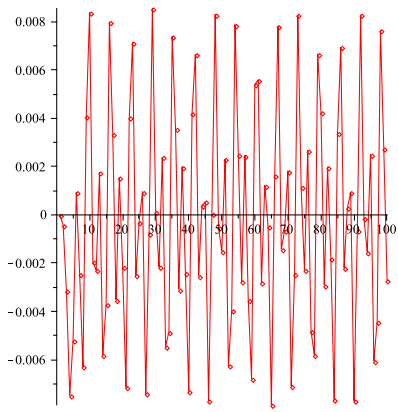

(e)

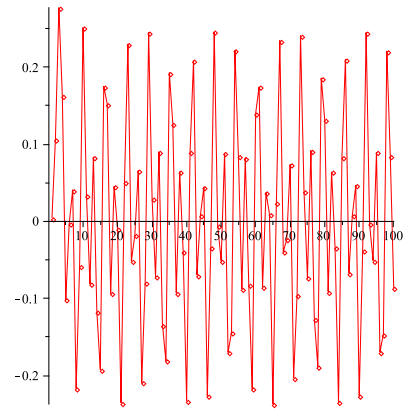

(c)



(f)

FiguRE 3.2. Scaled errors $\omega^{r+1}\left|\tilde{\boldsymbol{F}}_{s}[f, g]-\boldsymbol{I}[f, g]\right|$ for $f(x)=\mathrm{e}^{x}$, $g(x)=(2-x)^{-1},[a, b]=[-1,2]$, and six different Filon-type methods (3.11): (a) $r=2, \nu=2$; (b) $r=2, \nu=4 ; c_{2}=0, c_{3}=1$; (c) $r=3, \nu=2$; (d) $r=3, \nu=3, c_{2}=1$; (e) $r=3, \nu=4$, $c_{2}=0, c_{3}=1$; (f) $r=4, \nu=2$. In all these methods $\mu_{1}=\mu_{\nu}=r$; otherwise $\mu_{k}=1$.

Our first observation is that, even when the asymptotic and Filon-type methods employ exactly the same information (i.e., when $\nu=2, \mu_{1}=\mu_{2}$, and there are no internal nodes), the Filon-type method is more precise. This is clear when comparing Figures 3.1(a) and 3.1(b) with Figures 3.2(a) and 3.2(f), respectively. This is consistent with a comparison of asymptotic and Filon-type methods for integrals (1.1) and can break down when interpolation of $f$ by $\phi$ is of a poor quality (the Runge example); cf. Olv08.

Our second observation is again in line with the theory of Filon-type methods for 'classical' integrals (1.1). The addition of internal nodes leaves the asymptotic error $\mathcal{O}\left(\omega^{-r-1}\right)$ intact. Yet, at a small extra cost, it significantly lowers the amplitude of the error. This is apparent when comparing Figures 3.2 (a) and 3.2 (b) or Figures 3.2(c), 3.2(d) and 3.2(e). The intuitive reason is that addition of internal points makes the interpolation error smaller, and this is reflected in the error of the Filon-type method.

3.3. Computing the generalised oscillator. As long as $\theta^{\prime} \neq 0$, the scope of both asymptotic and Filon-type methods generalises at once to the integral $\boldsymbol{I}[f, g, \theta]$. In place of the asymptotic method (3.1) we truncate (2.5), while expressing each 
$\tilde{f}^{(k)}(x)$ for $x \in\{a, b\}$ as a linear combination of $f^{(i)}(x), i=0,1, \ldots, k$, with coefficients that depend upon $\theta$.

A generalisation of the Filon-type method to this setting is even simpler. We again choose nodes $a=c_{1}<c_{2}<\cdots<c_{\nu}=b$ and corresponding weights $\mu_{1}, \mu_{2}, \ldots, \mu_{\nu} \geq 1$. The function $\tilde{f}$ is interpolated by

$$
\tilde{\phi}(t)=\sum_{l=0}^{N} \phi_{l}\left[\theta^{-1}(t)\right]^{l} .
$$

Specifically, we require

$$
\tilde{\phi}^{(i)}\left(\theta\left(c_{k}\right)\right)=\tilde{f}^{(i)}\left(\theta\left(c_{k}\right)\right), \quad i=0, \ldots, \mu_{k}-1, \quad k=1,2, \ldots, \nu .
$$

However, since each $\tilde{f}^{(i)}(\theta(x))$ is a linear combination of $f(x), f^{\prime}(x), \ldots, f^{(i)}(x)$, it follows at once that (3.12) is equivalent to (3.7) where, again, $\phi$ is an $n$ th-degree algebraic polynomial. Therefore, in the absence of stationary points, a Filon-type method for (1.5) is identical to that for (1.4).

The computation of the generalised oscillator (1.5) in the presence of stationary points is made considerably more complicated by the presence of the term

$$
\zeta(\omega)=\int_{a}^{b} g(\sin [\omega \theta(x)]) \mathrm{d} x .
$$

In principle, we can expand $\zeta$ similarly to (2.1),

$$
\zeta(\omega)=\sum_{n=0}^{\infty} \frac{g^{(n)}(0)}{n !} \int_{a}^{b} \sin ^{n}[\omega \theta(x)] \mathrm{d} x,
$$

and proceed as in Subsection 2.1, but fairly rapidly we are faced by fairly unpleasant expressions. Although all of this can be accomplished, with a great deal of effort, for simple functions $\theta$, e.g., $\theta(x)=x^{q}$ for $q \in \mathbb{N}$, we see no point of embarking on such a long calculation without further motivation of a specific application.

Once $\zeta$ can be computed, we generalise our method along similar lines to the Filon-type method in the presence of stationary points in IN05. Thus, in addition to multiplicity- $r$ interpolation at the endpoint, we interpolate to multiplicity $(m+1) r$ at any stationary point $c$ of degree $m \geq 1$ (that is, $\theta^{(i)}(c)=0, i=$ $\left.1,2, \ldots, m, \theta^{(m+1)}(c) \neq 0\right)$ and perhaps at additional points.

\section{Discussion}

The main purpose of this paper has been to explore asymptotic and numerical features of a new model for composite highly oscillatory integrals. This model is of relevance in the simulation of electronic circuits, but we believe that its potential importance has a wider range and that it is of independent mathematical interest.

We have singled out Filon-type methods as the method of choice for our integrals. It might well have been possible to extend Levin-type methods to this setting. However, the remaining member of the triad of modern methods for highly oscillatory quadrature, the method of numerical steepest descent of [HV06, is probably of no use in the current situation. The idea underlying this approach is to integrate in the complex plane, along trajectories where the integral decays exponentially. This can be done very effectively with integrals of the form (1.1) but not, say, (1.3), since there is no trajectory $z(x+\mathrm{i} y)$ linking $a, \infty$, and $b$ in the complex plane along 
which $\sin [\omega z(x+\mathrm{i} y)]$ decays exponentially along the segments linking $a$ and $b$ to $\infty$.

Insofar as classical methods for the determination of asymptotic behaviour of integrals (the method of stationary phase, the method of steepest descent, and Perron's method; cf. Olv74, Won01]) are concerned, they are in general suitable for integrals of the form $\int f(x) \mathrm{e}^{\mathrm{i} \omega g(x)} \mathrm{d} x$, placing (1.4) outside their scope. The remedy is to use the methodology of Section 2 and consider infinite series in such integrals. This, however, is outside the scope of this paper, which is concerned with the numerical calculation of integrals of the form (1.4) through asymptotic analysis, rather than with asymptotic analysis per se.

\section{REFERENCES}

[AS64] M. Abramowitz and I. A. Stegun (eds.), Handbook of mathematical functions, National Bureau of Standards, Washington, DC, 1964. MR0167642 (29:4914)

$\left[\mathrm{CDI}^{+} 09\right]$ M. Condon, A. Deaño, A. Iserles, K. Maczyński, and T. Xu, On numerical methods for highly oscillatory problems in circuit simulation, COMPEL 28 (2009), 1607-1618. MR2597311

[CDI09] M. Condon, A. Deaño, and A. Iserles, On highly oscillatory problems arising in electronic engineering, M2AN 43 (2009), 785-804. MR2542877

[DCB05] E. Dautbegovic, M. Condon, and C. Brennan, An efficient nonlinear circuit simulation technique, IEEE Trans. Microwave Theory \& Techniques 53 (2005), 548-555.

[DR84] P. J. Davis and P. Rabinowitz, Methods of numerical integration, 2nd ed., Academic Press, Orlando, FL, 1984. MR760629 (86d:65004)

[HV06] D. Huybrechs and S. Vandewalle, On the evaluation of highly oscillatory integrals by analytic continuation, SIAM J. Num. Anal. 44 (2006), 1026-1048. MR2231854 (2007d:41033)

[IN04] A. Iserles and S. P. Nørsett, On quadrature methods for highly oscillatory integrals and their implementation, BIT 44 (2004), 755-772. MR2211043 (2006k:65060)

[IN05] _ Efficient quadrature of highly oscillatory integrals using derivatives, Proc. Royal Soc. A 461 (2005), 1383-1399. MR2147752 (2006b:65030)

[IN08] , From high oscillation to rapid approximation I: Modified Fourier expansions, IMA J. Num. Anal. 28 (2008), 862-887. MR2457350

[Lev96] D. Levin, Fast integration of rapidly oscillatory functions, J. Comput. Appl. Math. 67 (1996), 95-101. MR1388139 (97a:65029)

[Olv74] F. W. J. Olver, Asymptotics and special functions, Academic Press, New York, 1974. MR0435697 (55:8655)

[Olv06] S. Olver, Moment-free numerical integration of highly oscillatory functions, IMA J. Num. Anal. 26 (2006), 213-227. MR2218631(2006k:65064)

[Olv08] , Numerical approximation of highly oscillatory integrals, Ph.D. thesis, DAMTP, University of Cambridge, 2008.

[Rai60] E. D. Rainville, Special functions, Macmillan, New York, 1960. MR0107725 (21:6447)

[Ste93] E. M. Stein, Harmonic analysis: Real-variable methods, orthogonality, and oscillatory integrals, Princeton University Press, Princeton, NJ, 1993. MR.1232192 (95c:42002)

[Won01] R. Wong, Asymptotic approximations of integrals, SIAM, Philadelphia, 2001. MR.1851050 (2002f:41023)

Department of Applied Mathematics and Theoretical Physics, Centre for Mathematical Sciences, University of Cambridge, Wilberforce Road, Cambridge CB3 0WA, UNITED KINGDOM

E-mail address: ai@damtp.cam.ac.uk

School of Mathematical Sciences, Tel Aviv University, Ramat Aviv, Tel Aviv 69978, ISRAEL

E-mail address: levin@tau.ac.il 